\title{
PROMOVENDO A INTEGRALIDADE ATRAVES DA REESTRUTURAÇÃO DO NÚCLEO DO IDOSO DE UM HOSPITAL REGIONAL DE PERNAMBUCO
}

\author{
Vanessa de Barros e Silva Mazer ${ }^{1}$ \\ Fábio Gonçalves Viana Neto² \\ Angélica Barbosa Arruda Patriota ${ }^{3}$ \\ Kátia Goretti Veloso Lins ${ }^{4}$
}

resumo

Introdução: O crescimento da população idosa é um fenômeno observado mundialmente. Diante dessa realidade devem ser criadas ações

\footnotetext{
1 Fisioterapeuta, especialista em Gestão do cuidado pelo Programa de Residência Multiprofissional em Atenção Hospitalar com Ênfase em Gestão do Cuidado do Hospital Regional Dom Moura (HRDM). Fisioterapeuta do Núcleo do Idoso do HRDM. Av. Simoa Gomes s/n, CEP: 55296-000, Garanhuns/ PE, Brasil. E-mail: vanessamazer@gmail.com.

2 Fisioterapeuta, Mestre em Terapia Intensiva - Sobrati, plantonista da Unidade de Terapia Intensiva do HRDM. Av. Simôa Gomes s/n, CEP: 55296-000, Garanhuns/PE, Brasil. E-mail: fabioviananeto@ gmail.com.

3 Nutricionista, especialista em médico-cirúrgico pelo programa de residência Multiprofissional de Interiorização da Atenção à Saúde, nutricionista do Núcleo do Idoso do HRDM. Av. Simoa Gomes s/n, CEP: 55296-000, Garanhuns/PE, Brasil. E-mail: angélica.patriota@hotmail.com.

4 Psicóloga, mestre em Educação para o Ensino em Saúde - Faculdade Pernambucana de Saúde - IMIP, coordenadora do Núcleo do Idoso do HRDM. Av. Simoa Gomes s/n, CEP: 55296-000, Garanhuns/PE, Brasil. E-mail: katlins13@msn.com.
} 
e estratégias para enfrentamento das principais necessidades dessa população levando em consideração a integralidade. $\bigcirc$ presente estudo teve como objetivo promover uma melhor assistência aos usuários do Núcleo do Idoso de um Hospital Regional através da construção de um instrumento de avaliação multiprofissional, reorganização do fluxo dos atendimentos e criação da sala de espera como espaço para educação em saúde. Metodologia: Trata-se de uma pesquisa de intervenção baseada na metodologia de pesquisa-ação e teve como amostra os profissionais que compõem o Núcleo do Idoso do HRDM. A intervenção foi realizada em três etapas: a elaboração do instrumento de avaliação multiprofissional, estruturação do fluxo de atendimentos e criação da sala de espera. Resultados e discussão: $\bigcirc$ instrumento de avaliação foi classificado como satisfatório pelos profissionais reforçando a importância desse tipo de ferramenta. Ao todo foram realizadas 11 salas de espera abordando diversos temas. As intervenções utilizadas para estruturação do fluxo de atendimentos favoreceram a continuidade e direcionalidade das ações interferindo diretamente a qualidade do serviço ofertado ao usuário. Conclusão: o presente estudo alcançou o objetivo proposto através da criação do instrumento de avaliação, da estruturação do fluxo de atendimentos e da criação da sala de espera. A elaboração de estratégias para garantir um olhar integral à saúde do idoso é um desafio e a utilização das tecnologias em saúde surge com o intuito de promover uma melhor assistência ao usuário.

palavras-chave

Envelhecimento. Saúde do Idoso. Integralidade em Saúde. Educação em Saúde. Assistência Centrada no Paciente.

O crescimento da população idosa é um fenômeno observado mundialmente e este se deve ao processo de transição demográfica e epidemiológica (DA CRUZ; CAETANO; LEITE, 2010; FERREIRA; YOSHITOME, 2010). No Brasil, essas transições iniciaram por volta de 1940, devidas principalmente à substituição das doenças transmissíveis por doenças não transmissíveis e o predomínio da morbidade ao invés da mortalidade (CHAIMOWICZ, 2009). Segundo análise realizada pelo Instituto Brasileiro de Geografia e Estatística (IBGE) 
em 2016, a população idosa representa 11,5\% da população total brasileira, sendo o sexto país do mundo em taxa de envelhecimento populacional (DA CRUZ; CAETANO; LEITE, 2010). Diante deste quadro e tendo em vista o crescimento da população idosa no Brasil, foi criada a Política Nacional de Saúde do Idoso em 1999, através da Portaria n. . 1395, sendo reformulada em 2006 através da Portaria n. 2528.

A criação de ações e estratégias para enfrentamento das principais necessidades da população idosa se configura como um desafio denso e árduo, pois a desarticulação do sistema dificulta uma lógica de assistência pautada na integralidade (DA CRUZ; CAETANO; LEITE, 2010; MENDES, 2012). As equipes de saúde devem estar preparadas para atuar de maneira tal que consigam identificar as necessidades do idoso de uma forma integral a fim de oferecer o cuidado essencial. Para isso, é necessário entender que as complexidades de suas demandas vão além de complicações crônicas e agudas, o que exige acompanhamento contínuo de uma equipe multiprofissional de saúde (REMOR et al., 2011).

Pensando em estratégias para efetivação da Política Nacional de Saúde do Idoso (BRASIL, 2017), a Secretaria de Saúde do Estado de Pernambuco criou em agosto de 2016, o Núcleo do Idoso no Hospital Regional Dom Moura (HRDM), no município de Garanhuns/PE. Esse equipamento é de fundamental importância para a V Região de Saúde, pois funciona como referência para os 21 municípios que dela fazem parte. De acordo com o DATASUS em senso realizado no ano de 2012 a população de idosos da V Regional é de 61.651, representando 11,89\% da população total dessa região (BRASIL, 2012).

O Núcleo do Idoso surgiu com o objetivo promover um serviço qualificado e multiprofissional composto por médico, enfermeira, fisioterapeuta, nutricionista, assistente social e psicóloga. Os atendimentos acontecem uma vez por semana e os idosos são acompanhados por toda a equipe multiprofissional. Para ingressar no Núcleo o idoso precisa ter idade maior que 65 anos e apresentar pelo menos três comorbidades. Desde a sua implantação até o mês de fevereiro do ano corrente, já foram realizados cerca de 357 atendimentos das categorias supracitadas, e, no momento, 136 idosos são acompanhados.

Diante disso, o presente estudo teve como objetivo contribuir para uma melhor assistência aos usuários do Núcleo do Idoso do HRDM, através da construção de um instrumento de avaliação multiprofissional, reorganização do fluxo dos atendimentos e criação da sala de espera como espaço para educação em saúde. 
O estudo foi realizado no Núcleo do Idoso do HRDM. Trata-se de um projeto de intervenção baseado na metodologia de pesquisa-ação. Esse tipo de metodologia tem como principal característica a aproximação do pesquisador e dos profissionais envolvidos com o objetivo de transformar alguma realidade (BALDISSERA, 2001). A presente pesquisa foi aprovada pelo Comitê de Ética da Autarquia Educacional de Belo Jardim (CAAE: 79946217.1.0000.5189 e Parecer n..: 2.470.504). A amostra do estudo foi formada pelos profissionais que compõe o Núcleo do Idoso do HRDM e como critério de exclusão os profissionais que se recusassem a participar da pesquisa. A intervenção foi realizada em três etapas, conforme fluxograma da Figura 1.

\section{Figura 1 - Fluxograma das etapas do estudo}

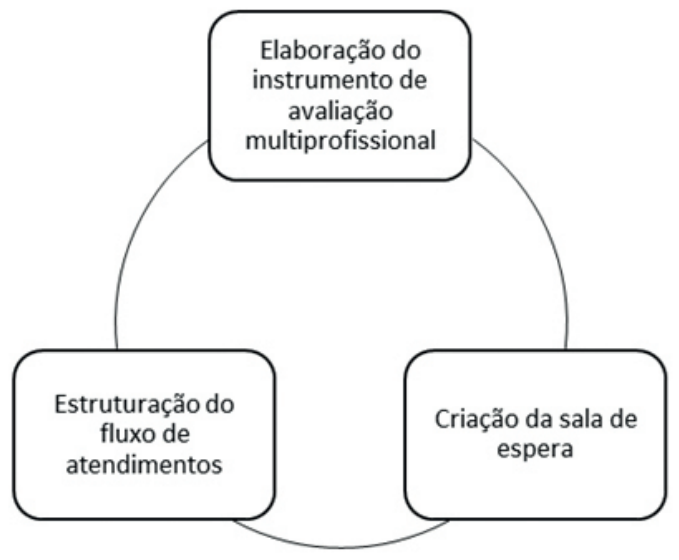

Fonte: Elaborada pelos autores.

Todas as etapas ocorreram de forma concomitante e para realização das mesmas foram realizadas reuniões com a equipe do Núcleo, encontros individuais com os profissionais e levantamento bibliográfico. As reuniões com toda a equipe foram planejadas para serem realizadas mensalmente bem como os encontros individuais com os profissionais. As ações da sala de espera, momento em que os usuários aguardam os atendimentos dos profissionais, foram avaliadas através da mensuração da quantidade de ações que deveriam ter sido realizadas e as que de fato foram realizadas. A avaliação do instrumento 
de avaliação multiprofissional e da estruturação do fluxo foi realizada através de um questionário de satisfação direcionado aos profissionais.

Esse questionário foi construído baseado no modelo de verificação de Likert. O mesmo contendo seis afirmativas (quatro referentes ao instrumento de avaliação e dois referentes ao fluxo): 1 . O instrumento de avaliação é de fácil preenchimento; 2. A linguagem utilizada no instrumento é clara e objetiva; 3. O conteúdo do instrumento de avaliação é satisfatório; 4 . O instrumento de avaliação é útil na rotina da minha prática profissional; 5 . A utilização do guia de atendimentos ajudou a melhorar o fluxo e 6 . A presença de um profissional guiando os usuários ajudou a melhorar o fluxo. Os dados coletados foram avaliados por meio de análise descritiva, que, segundo Silvestre (2007), é utilizada com o objetivo de organizar e descrever os dados. Para isso, foram construídos gráficos, tabelas e fluxogramas, através do programa Microsoft Excel 2010.

\section{Resultados e discussão}

A pesquisa foi realizada no período de setembro de 2017 a fevereiro de 2018. Todos os integrantes do Núcleo aceitaram participar, totalizando uma amostra de seis indivíduos (um médico, uma enfermeira, um fisioterapeuta, uma psicóloga, um assistente social e uma nutricionista). Inicialmente, foi realizada uma reunião com a equipe multiprofissional objetivando uma explanação e discussões sobre as intervenções. Esse momento serviu como um espaço para a promoção de uma integração inicial entre o pesquisador e os profissionais. Além disso, elencaram-se discussões a respeito da pesquisa e qual a melhor maneira de conduzi-la de forma que fosse uma intervenção relevante para o grupo.

Para a efetivação de todas as etapas foram realizadas reuniões entre a equipe e o pesquisador e encontros individuais com os profissionais do Núcleo. Entretanto, houve dificuldades em realizar reuniões de forma periódica e conseguir a participação de toda a equipe, devido à limitação da disponibilidade de alguns integrantes do grupo. Durante os seis meses do estudo, houve quatro reuniões entre a equipe, contudo, nenhuma teve a presença de todos os profissionais.

Em estudo realizado por Peduzzi et al. (2011), foi demonstrada a dificuldade na realização de reuniões de equipe, principalmente devido aos diferentes turnos de trabalho dos profissionais e à pouca disponibilidade de tempo. A presente pesquisa corrobora com os achados do autor supramencionado, onde 
foi possível perceber que a rotina sobrecarregada e as diversas atribuições que os profissionais possuíam dificultaram a continuidade desses espaços de partilha de conhecimentos e construção de mudanças. Esses espaços de encontro entre os profissionais devem ter como objetivo o planejamento para se alterar alguma realidade. Segundo Campos, Faria e Santos (2010), o planejamento surge através da necessidade de se alcançar algum objetivo e deve ser realizado principalmente quando se almeja um propósito complexo que precisa da interação de diferentes áreas profissionais, como é o caso do Núcleo do Idoso.

Para criação da sala de espera, momento em que os usuários esperam para serem atendidos, foi construído um cronograma junto à equipe com a sequência dos profissionais que realizariam as salas durante os dias de atendimento em cada mês. Esse cronograma foi seguido durante os seis meses e no total ocorreram 11 salas de espera, 100\% do que havia sido planejado, com uma média de 9,4 participantes por sala, como mostra a Tabela 1.

Tabela 1 - Ações de educação em saúde na sala de espera do Núcleo do ldoso do Hospital Regional Dom Moura durante o ano de 2017 e 2018

\begin{tabular}{|c|c|c|c|}
\hline Data & Tema & $\begin{array}{l}\text { Profissional } \\
\text { responsável }\end{array}$ & $\begin{array}{l}\text { Número de } \\
\text { participantes }\end{array}$ \\
\hline 10/11/2017 & Benefício de Prestação Continuada & $\begin{array}{l}\text { Assistente } \\
\text { Social }\end{array}$ & 9 \\
\hline $17 / 11 / 2017$ & $\begin{array}{l}\text { Mitos e verdades sobre alimentação } \\
\text { saudável }\end{array}$ & Nutricionista & 6 \\
\hline $27 / 11 / 2017$ & $\begin{array}{l}\text { Importância do exercício e suas } \\
\text { particularidades }\end{array}$ & Fisioterapeuta & 7 \\
\hline $01 / 12 / 2017$ & Autocuidado e qualidade de vida & Psicóloga & 10 \\
\hline 08/12/2017 & Importância da hidratação & Nutricionista & 9 \\
\hline 15/12/2017 & Violência contra o idoso & $\begin{array}{l}\text { Assistente } \\
\text { Social }\end{array}$ & 15 \\
\hline 22/12/2017 & Orientações ergonômicas & Fisioterapeuta & 12 \\
\hline 02/02/2018 & $\begin{array}{l}\text { Depressão, isolamento, solidão e falta de } \\
\text { cuidado consigo mesmo para uma quali- } \\
\text { dade de vida }\end{array}$ & Psicóloga & 12 \\
\hline 09/02/2018 & Carnaval & Nutricionista & 7 \\
\hline 16/02/2018 & Envelhecimento & $\begin{array}{l}\text { Assistente } \\
\text { Social }\end{array}$ & 7 \\
\hline 23/02/2018 & $\begin{array}{l}\text { Artrite, artrose e osteoporose: particulari- } \\
\text { dades e maneiras de aliviar os sintomas }\end{array}$ & Fisioterapeuta & 10 \\
\hline
\end{tabular}


A sala de espera se configura como um espaço de reflexão que estimula a proximidade da população ao serviço, provocando a construção de dúvidas, críticas e opiniões sobre diversos assuntos inclusive relacionados ao envelhecimento e para tal deve-se utilizar de metodologias que introduzam o usuário em todo o processo (ASSIS et al., 2007; COMBINATO et al., 2010). Durante a realização das salas de espera desta pesquisa, os profissionais utilizaram metodologias ativas a fim de estimular a participação de todos. Esse tipo de metodologia é caracterizado pela utilização da problematização como estratégia para aprendizagem, fazendo com que o indivíduo reflita e possa ressignificar suas descobertas e assim exercitar a liberdade e autonomia na realização de escolhas (FREITAS et al., 2015). Durante esses momentos foi observado como essa população necessita de ações de educação em saúde, pois apresentam demandas complexas que muitas vezes são identificadas durante esses momentos, como, por exemplo, a presença de mais de uma comorbidade de saúde junto à vulnerabilidade social, relações fragilizadas dentro de suas residências e com a rede de saúde, falta de conhecimento acerca de seus direitos, entre outros. Diante desse contexto, a presença de uma equipe multiprofissional torna-se indispensável.

A Tabela 1 mostra os temas que foram abordados nas salas de espera durante os seis meses do estudo e esses resultados entram em consonância com pesquisa realizada por Mallmann e colaboradores (2015), que trazem como principais temas a serem abordados para educação em saúde com idosos a promoção da saúde principalmente através da realização de exercícios físicos, alimentação saudável e orientações quanto ao apoio social. Apesar da maioria das salas de espera terem ocorrido com um tema já predefinido é importante destacar que muitas vezes durante a sua realização o tema foi moldado a partir das demandas que surgiam dos usuários. Observamos ainda na Tabela 1 que as salas de espera foram realizadas por quatro categorias de profissionais e a equipe era comporta por seis. Tal fato ocorreu devido às limitações de disponibilidade destes profissionais por conta dos seus atendimentos nos dias que ocorreram as salas de espera.

Para além dos resultados quantitativos, observamos as reações dos idosos durante esses momentos. No decorrer de todas as salas os usuários se mostraram interessados nas temáticas e contribuíram de forma ativa para a realização das mesmas. Relataram a importância desses momentos para seu processo de envelhecimento, pois foi possível a aquisição de novos conhecimentos através das trocas de experiências vivenciadas. Desenvolvida a partir da identificação de problemas do cotidiano profissional, a educação permanente procura levar em consideração os conhecimentos e as experiências pré-existentes das 
equipes e dos usuários, além do conhecimento técnico, com o objetivo de transformar as práticas em saúde (LOPES et al., 2007). Esse dispositivo funciona aproximando o cotidiano do profissional do SUS com as necessidades da população promovendo a construção de políticas locais e formação e produção de conhecimentos necessários para formulação de políticas públicas de saúde, favorecendo o poder deliberativo da população através do fortalecimento de sua participação (LOPES et al., 2007).

Durante a elaboração do instrumento de avaliação multiprofissional (Apêndice A), foram discutidos aspectos relevantes para cada profissão, que deveriam estar contidos no mesmo, como, por exemplo, escalas de avaliação de funcionalidade e de depressão. Para construção do instrumento cada categoria profissional elaborou um trecho e, no fim, eles foram sintetizados em um único instrumento e assim durante o processo de avaliação cada um preenchia sua parte e tinha acesso às informações dos outros profissionais. Após a construção do instrumento, baseado nos domínios preconizados pela Organização Pan-Americana da Saúde (MORAES, 2012) e com a contribuição de todas as categorias profissionais, ele foi apresentado à toda equipe do Núcleo do Idoso, através de uma reunião, para apreciação de todos e possíveis alterações, e em seguida passou a ser utilizado durante os atendimentos aos usuários.

Para avaliação desse instrumento os profissionais responderam um questionário de satisfação construído de acordo com a escala de Likert, onde foi atribuída uma pontuação para cada alternativa: um ponto para "discordo totalmente", dois pontos para "discordo parcialmente", três pontos para "não concordo nem discordo", quatro pontos para "concordo parcialmente" e cinco pontos para "concordo totalmente".

A Figura 2 mostra o gráfico com a média das respostas dos profissionais referentes a cada item do questionário de avaliação. Pode-se observar que nos itens referentes ao preenchimento e conteúdo do instrumento (questões 1 e 3), a maioria das respostas ficou entre "concordo parcialmente" e "concordo totalmente", já os itens referentes à linguagem e à contribuição da ficha para a prática profissional (questões 2 e 4) apresentaram a pontuação máxima referente a "concordo totalmente". Esses resultados demonstram que houve um grau de satisfação positivo dos profissionais em relação ao instrumento. 
Figura 2 - Gráfico referente à média da resposta dos profissionais para o Questionário de Avaliação do Instrumento criado

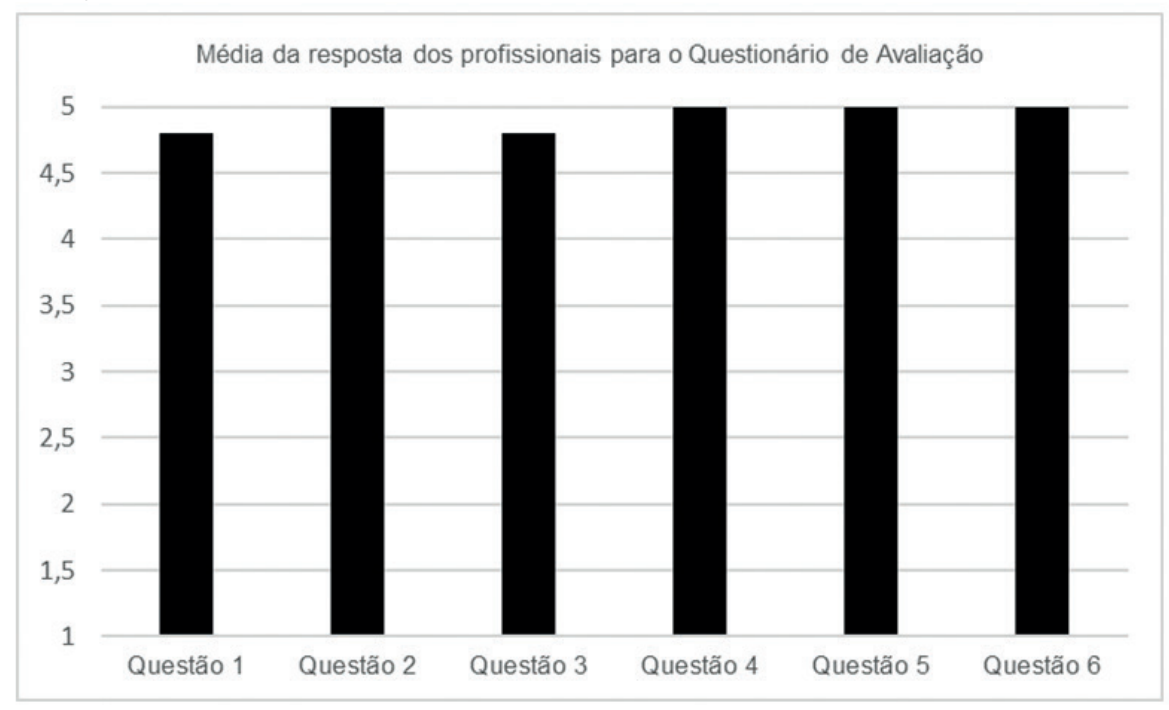

Fonte: Elaborada pelos autores.

A avaliação multiprofissional do idoso é uma ferramenta importante, pois considera os múltiplos diagnósticos em saúde a que os idosos estão sujeitos e descobre problemas que geralmente não são abordados de forma adequada, necessitando do envolvimento dos profissionais, do idoso e da família (MORAES, 2012; RODRIGUES et al., 2015). Durante a construção do instrumento de avaliação, os profissionais do Núcleo tiveram a oportunidade de adequá-lo à realidade de suas rotinas elaborando uma ficha de fácil preenchimento que pudesse diagnosticar e quantificar as dificuldades dos idosos para, a partir disso, estabelecer um planejamento individualizado.

A demanda da estruturação do fluxo surgiu a partir da necessidade de se organizar os atendimentos da equipe multiprofissional para que os idosos fossem atendidos por todos da equipe. Para isso, foi criado um Guia de Atendimentos Individual contendo o nome de todas as categorias profissionais que atendem no Núcleo e esses nomes eram destacados à medida que o usuário fosse atendido por cada categoria. Dessa forma era possível saber quais profissionais atenderam o idoso e quais ainda faltavam. Associado a isso, a pesquisadora auxiliou o fluxo através do acompanhamento desses Guias junto aos usuários, pois foi observado e pontuado pelos profissionais a necessidade de uma pessoa de apoio para garantir o seguimento do mesmo. 
Os resultados referentes às respostas dos profissionais em relação às intervenções realizadas para organizar o fluxo de atendimentos estão demonstrados na Figura 2. Todos os profissionais pontuaram concordo totalmente para as duas questões referentes ao fluxo (questões 5 e 6) afirmando que a utilização do guia de atendimentos e a presença de um profissional guiando os usuários ajudou a melhorar o fluxo.

Roese e Gerhardt (2008) definem fluxos de utilização de serviços como uma busca por um atendimento ou a trajetória percorrida pelo usuário, estando diretamente relacionado a todos os esforços realizados para se conseguir uma resolutividade. $\mathrm{O}$ emprego de instrumentos que favoreçam os fluxos garante a direcionalidade e continuidade das ações interferindo diretamente na qualidade do serviço ofertado ao usuário além de melhorar os processos de trabalho (WERNECK; FARIA; CAMPOS, 2009). Durante as intervenções para o fluxo foi possível observar a participação de todos os profissionais nesse processo e uma consequente melhoria da qualidade dos atendimentos por conta da sua organização.

Segundo Oliveira e Cutolo (2012), a integralidade não significa totalidade quando levamos em consideração a complexidade do processo saúde-doença, e se configura no momento em que os profissionais estão em contato com os usuários, interagindo e procurando um território em comum com o objetivo de favorecer o contato com o mesmo. Esse princípio não exclui o conhecimento sobre as doenças, mas proporciona uma visão mais abrangente sobre as necessidades do sujeito, sendo realizada quando se procura um estabelecimento de uma relação com o usuário promovendo um cuidar compromissado, baseado em responsabilidade e confiança (OLIVEIRA; CUTOLO, 2012).

\section{Considerações finais}

A criação de estratégias para garantir a integralidade da atenção à saúde para o idoso é um desafio, o presente estudo alcançou o objetivo proposto através da criação de um instrumento de avaliação multidisciplinar do idoso, da estruturação do fluxo de atendimentos e da criação da sala de espera. A partir dessas intervenções foi possível observar uma melhora na qualidade nos atendimentos dos usuários no Núcleo do Idoso. Estudos que utilizam a metodologia da pesquisa-ação são de extrema importância, pois possuem a capacidade de mudar alguma realidade e para isso deve haver o comprometimento de todos os envolvidos. Durante a execução do trabalho, pode-se pontuar como uma limitação a dificuldade, em alguns momentos, do compro- 
metimento de todos os profissionais, como foi discutido anteriormente no tocante as reuniões.

O cuidado segundo Cecílio e Merhy (2003, p. 2) "é um somatório de pequenos cuidados parciais que vão se complementando, de maneira mais ou menos consciente e negociada, entre os vários cuidadores" e para geri-lo é necessário a utilização das tecnologias de saúde (leves, leve-duras e duras). Durante a pesquisa, foi possível observar a utilização dessas tecnologias com o objetivo de promover uma melhor assistência ao usuário a partir de um olhar pautado no cuidado integral.

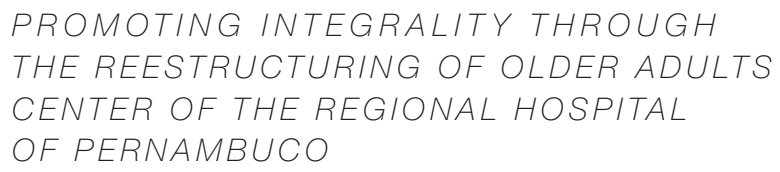

abstract

Introduction: The growth of the older adult population is a phenomenon observed worldwide. For this reason, must be applied actions and strategies to overcome the main necessities of this population, considering the principle of completeness. This study aimed promoting a better user assistance of Older Adult Center of a Regional Hospital through the construction of a multi professional evaluation system, reorganizing the attendance flow and creating a waiting room as a health education space. Materials and methods: This research is about an intervention based on research-action methodology and had as sample the professionals working on the HRDM's Older Adult Center. This intervention occurred in 3 steps: the multi professional evaluation system elaboration, flow attendance structuring and waiting room creation. Results and discussion: The evaluation instrument was measured as satisfactory by the professionals, reinforcing the importance of this tool. Altogether, it was accomplished 11 waiting rooms about various subjects. The interventions used to structured the attendance flow benefited the action's continuity and directionality, interfering directly in the quality of the service offered to the user. Conclusion: the present study, as well as the proposed objective through the creation of the evaluation tool, the structuring of care and the creation of the waiting room. An elaboration of strategy to guarantee an integral look at the health of older adults is a challenge, 
and the use of health technologies comes forward with the purpose of promoting the improvement of the user.

keywords

Aging. Health of Older Adults. Integrality in Health. Health Education. Patient-Centered Care.

referências

ASSIS, Mônica de et al. Ações educativas em promoção da saúde no envelhecimento: a experiência do núcleo de atenção ao idoso da UNATI/UERJ. O Mundo da Saúde, São Paulo, v. 31, n. 3, p. 438-447, jul./set. 2007.

BALDISSERA, Adelina. Pesquisa-ação: uma metodologia do "conhecer" e do "agir" coletivo. Sociedade em Debate, Rio Grande do Sul, v. 7, n. 2, p. 5-25, ago. 2001.

BRASIL. Portaria n. ${ }^{\circ}$.528 de 19 de outubro de 2006. Aprova a Política Nacional de Saúde da Pessoa Idosa. Brasília, DF: Ministério da Saúde, 2006. Disponível em: http:// bvsms.saude.gov.br/bvs/saudelegis/gm/2006/prt2528_19_10_2006.html. Acesso em: 5 set. 2017.

BRASIL. Ministério da Saúde. DATASUS: TABNET. Brasília, DF: Ministério da Saúde, 2012. Disponível em: http://tabnet.datasus.gov.br/cgi/deftohtm.exe?ibge/cnv/poppe. def.2012. Acesso em: 3 set. 2017.

CAMPOS, Francisco Carlos Cardoso de; FARIA, Horácio Pereira de; SANTOS, Max André dos. Planejamento e avaliação das ações em saúde. 2. ed. Belo Horizonte: Núcleo de Educação em Saúde Coletiva da Faculdade de Medicina/UFMG, 2010.

CECÍlIO, Luiz Carlos de Oliveira; MERHY, Elias Emerson. A integralidade do cuidado como eixo da gestão hospitalar. Campinas: UNICAMP, 2003. Mimeografado.

CHAIMOWICZ, Flávio et al. Saúde do idoso. Belo Horizonte: Núcleo de Educação em Saúde Coletiva da Faculdade de Medicina UFGM, 2009.

COMBINATO, Denise Stefanoni et al. "Grupos de conversa": saúde da pessoa idosa na Estratégia Saúde da Família. Psicologia \& Sociedade, Minas Gerais, v. 22, n. 3, p. 558-568, 2010.

DA CRUZ, Danielle Teles; CAETANO, Vanusa Caiafa; LEITE, Isabel Cristina Gonçalves. Envelhecimento populacional e bases legais da atenção à saúde do idoso. Caderno de Saúde Coletiva, Rio de Janeiro, v. 18, n. 4, p. 500-508, 2010.

FERREIRA, Denise Cristina de Oliveira; YOSHITOME, Aparecida Yoshie. Prevalência e caraterísticas das quedas de idosos institucionalizados. Revista Brasileira de Enfermagem, São Paulo, v. 63, n. 6, p. 991-997, 2010.

FREITAS Maria Cilene et al. Uso de metodologias ativas de aprendizagem para educação na saúde: análise da produção científica. Trabalho, Educação e Saúde, Rio de Janeiro, v. 13, p. 117-130, 2015.

INSTITUTO BRASILEIRO DE GEOGRAFIA E ESTATÍSTICA (IBGE). Coordenação de População e Indicadores Sociais. Síntese de indicadores sociais: uma análise das condições de vida da população brasileira: 2016. Rio de Janeiro: IBGE, 2016. ISSN 1516-3296. n. 36. 
LOPES, Sara Regina Souto et al. Potencialidades da educação permanente para a transformação das práticas de saúde. Comunicação Ciências Saúde, Brasília, v. 18, n. 2, p. 147-155, 2007.

MALLMANN, Danielli Gavião et al. Educação em saúde como principal alternativa para promover a saúde do idoso. Ciência \& Saúde Coletiva, Recife, v. 20, n. 6, p. 1763-1772, jun. 2015 .

MENDES, Antônio da Cruz Gouveia. Assistência pública de saúde no contexto da transição demográfica brasileira: exigências atuais e futuras. Caderno de Saúde Pública, Rio de Janeiro, v. 28, n. 5, p. 955-964, 2012.

MORAES, Edgar Nunes de. Atenção à saúde do idoso: aspectos conceituais. Brasília, DF: Organização Pan-Americana da Saúde, 2012.

OLIVEIRA, Inajara Carla; CUTOLO, Luiz Roberto Agea. Humanização como expressão de integralidade. Mundo Saúde, São Paulo, v. 36, n. 3, p. 502-506, 2012.

PEDUZZI, Marina et al. Trabalho em equipe na perspectiva da gerência de serviços de saúde: instrumentos para a construção da prática interprofissional. Physis Revista de Saúde Coletiva, Rio de Janeiro, v. 21, p. 629-646, 2011.

REMOR, Camila Bitencourt et al. Ambulatório multiprofissional de geriatria: uma perspectiva de assistência à saúde do idoso na busca da interdisciplinaridade. Revista Brasileira de Ciências do Envelhecimento Humano, Passo Fundo, v. 8, n. 3, set./dez. 2011.

RODRIGUES, Rogério Manuel Clemente et al. Os muito idosos: avaliação funcional multidimensional. Revista de Enfermagem Referência, Coimbra, v. 5, n. 4, p. 65-74, jun. 2015.

ROESE, Adriana; GERHARDT, Tatiana Engel. Fluxos e utilização de serviços de saúde: mobilidade dos usuários de média complexidade. Revista Gaúcha de Enfermagem, Porto Alegre, v. 29, n. 2, p. 221-229, jun. 2008.

SILVESTRE, Antonio Luiz. Análise de Dados e Estatística Descritiva. 1. ed. Portugal: Escolar Editora, 2007.

WERNECK, Marcos Azeredo Furquim; FARIA, Horácio Pereira de; CAMPOS, Kátia Ferreira Costa. Protocolos de cuidado à saúde e de organização do serviço. Belo Horizonte: Núcleo de Educação em Saúde Coletiva da Faculdade de Medicina/UFGM, 2009. 
Data:

Cartão SUS:

1. Dados gerais:

Nome:

Idade: Data de nascimento: Sexo: ( ) Masc. ( ) Fem.

Endereço: Telefone:

Estado civil: ( ) Casado(a) ( ) Solteiro(a) ( ) Divorciado(a) ( ) Viúvo(a)

2. Queixa principal:

\section{História Pregressa:}

Comorbidades associadas:

Tabagismo: Sim ( ) Não ( )

Etilismo: Sim ( ) Não ( )

Carga tabágica:

Atividade física regular: Sim ( ) Não ( )

Vacinação atual: Influenza ( ) DPT ( ) Pneumonia ( ) HBV ( ) Varicela-Zoster ( ) Hospitalização prévia: Sim ( ) Não ( )

Cirurgias prévias: $\operatorname{Sim}($ ) Não ( )

Hemotransfusão: $\operatorname{Sim}($ ) Não ( )

Alergias: Sim ( ) Não ( )

Reações adversas a medicação: $\operatorname{Sim}($ ) Não ( )

Doenças na família:

\section{Sintomas cardiovasculares e respiratórios:}

\section{Sintomas genitourinários:}


6. Sintomas neurológicos:

7. Sintomas do aparelho digestório:

8. Sintomas osteoarticulares:

9. Lista de medicamentos

\begin{tabular}{|l|l|l|}
\hline Droga & Dose & Tempo de uso \\
\hline & & \\
\hline & & \\
\hline & & \\
\hline & & \\
\hline & & \\
\hline & & \\
\hline & & \\
\hline & & \\
\hline
\end{tabular}

10. Exames bioquímicos: 


\section{Sistematização da enfermagem:}

Forma de locomoção: ( ) Cadeira de rodas ( ) Deambula com ajuda ( ) Deambula sozinho ( ) Uso de bengala/muleta Pressão Arterial: Glicemia:

Respiração: ( ) Normal ( ) Dispneia ( ) Dispneia aos esforços Olhos: ( ) Óculos ( ) Edema ( ) Secreção ( ) Diplopia ( ) Prurido ( ) Turvação Ouvidos: ( ) Dor ( ) Secreção ( ) Dificuldade auditiva ( ) Zumbidos ( ) Uso de aparelho auditivo

Pele: ( ) Hidratada ( ) Desidratada ( ) Fria ( ) Hiperemia ( ) Turgor alterado ( ) Cianose ( ) Edema ( ) Lesão

Evidenciados por: ( ) Equimose ( ) Perda de pelos ( ) Erupção cutânea ( ) Veias varicosas ( ) Outros:

Estado dos músculos esqueléticos: ( ) Tremores ( ) Fraqueza

Dentadura: ( ) Não ( ) Sim: ( ) Superior ( ) Inferior

TGI: ( ) Dificuldade para mastigar ( ) Disfagia ( ) Azia ( ) Náusea ( ) Êmese ( ) Afta ( ) Constipação ( ) Diarreia ( ) Outros

Genitourinário: ( ) Disúria ( ) Incontinência ( ) Uso de SVD ( ) Cistostomia ( ) Uso de SVA ( ) Leucorreia ( ) Atrofia Vaginal ( ) Sangramentos ( ) Aumento de próstata ( ) Prolapso uterino ( ) Cistocele ( ) Lesões

Mamas: ( ) Dor ( ) Edema ( ) Hiperemia ( ) Nódulos ( ) Líquido a expressão

\section{DIAGNÓSTICOS:}

( ) Fragilidade emocional

( ) Deambula com dificuldade

( ) Não consegue dormir

( ) Não consegue de alimentar

( ) Aumento da diurese

( ) Sede excessiva

( ) Sono excessivo

( ) Dor em MMII

( ) Descamação do couro cabeludo

( ) Lábios ressecados

( ) Precariedade na dentição

( ) Alteração pressórica

( ) Hipoglicemia

( ) Hiperglicemia

\section{Exame mental - Psicologia:}




\section{Escala de Depressão Geriátrica Abreviada (GDS-15)* - Psicologia:}

\begin{tabular}{|c|c|c|}
\hline & Pergunta & Pontuação \\
\hline 1 & Está satisfeito(a) com sua vida? (não $=1)(\operatorname{sim}=0)$ & \\
\hline 2 & Interrompeu muitas de suas atividades? $(\operatorname{sim}=1)($ não $=0)$ & \\
\hline 3 & Acha sua vida vazia? $(\operatorname{sim}=1)($ não $=0)$ & \\
\hline 4 & Aborrece-se com frequência? $(\operatorname{sim}=1)($ não $=0)$ & \\
\hline 5 & $\begin{array}{l}\text { Sente-se bem com a vida na maior parte do tempo? }(\text { não }=1) \\
(\operatorname{sim}=0)\end{array}$ & \\
\hline 6 & Teme que algo ruim lhe aconteça? $(\operatorname{sim}=1)($ não $=0)$ & \\
\hline 7 & Sente-se alegre a maior parte do tempo? $($ não $=1)(\operatorname{sim}=0)$ & \\
\hline 8 & Sente-se desamparado com frequência? $(\operatorname{sim}=1)($ não $=0)$ & \\
\hline 9 & $\begin{array}{l}\text { Prefere ficar em casa a sair e fazer coisas novas? }(\operatorname{sim}=1) \\
(\text { não }=0)\end{array}$ & \\
\hline 10 & $\begin{array}{l}\text { Acha que tem mais problemas de memória que outras pessoas? } \\
(\operatorname{sim}=1)(\text { não }=0)\end{array}$ & \\
\hline 11 & Acha que é maravilhoso estar vivo(a)? (não $=1)(\operatorname{sim}=0)$ & \\
\hline 12 & Sente-se inútil? $($ não $=0)(\operatorname{sim}=1)$ & \\
\hline 13 & Sente-se cheio/a de energia? $($ não $=1)(\operatorname{sim}=0)$ & \\
\hline 14 & Sente-se sem esperança? $($ não $=0)(\operatorname{sim}=1)$ & \\
\hline 15 & Acha que os outros têm mais sorte que você? $(\operatorname{sim}=1)($ não $=0)$ & \\
\hline & TOTAL & \\
\hline
\end{tabular}

*Escala adaptada da GDS 30 de Sheikh e Yesavage.

*Questões que pontuam $\operatorname{sim}(2,3,4,6,8,9,10,15)$.

*Questões que pontuam não $(1,5,7,11,12,13,14)$.

\section{CLASSIFICAÇÃO:}

( ) 0 -5: Normal

( ) 6 -10: Depressão leve

( ) $11-15$ : Depressão severa

\section{Funcionalidade (Índice de Katz) - Fisioterapia:}

\begin{tabular}{|c|c|c|}
\hline \multicolumn{3}{|c|}{$\begin{array}{l}\text { Para cada área de funcionamento listada abaixo assinale a descrição que melhor se aplica. A palavra "assistência" } \\
\text { significa supervisão, orientação ou auxílio pessoal }\end{array}$} \\
\hline \multicolumn{3}{|c|}{ Banho - banho no leito, banheira ou chuveiro } \\
\hline $\begin{array}{c}\text { Não recebe assistência (entra e sai da } \\
\text { banheira sozinho se essa é } \\
\text { usualmente utilizada para banho) }\end{array}$ & $\begin{array}{l}\text { Recebe assistência no banho } \\
\text { somente para uma parte do corpo } \\
\text { (como costas ou uma perna) }\end{array}$ & $\begin{array}{l}\text { Recebe assistência no banho em } \\
\text { mais de uma parte do corpo }\end{array}$ \\
\hline \multicolumn{3}{|c|}{ Vestir - pega roupa no armário e veste, incluindo roupas íntimas, roupas externas e fechos e contos (caso use) } \\
\hline $\begin{array}{l}\text { Pega roupas e se veste } \\
\text { completamente sem assistência }\end{array}$ & $\begin{array}{l}\text { Pega roupas e se veste sem } \\
\text { assistência, exceto para amarrar os } \\
\text { sapatos }\end{array}$ & $\begin{array}{c}\text { Recebe assistência para pear as } \\
\text { roupas ou para vestir-se ou } \\
\text { permanece parcial ou totalmente } \\
\text { despido }\end{array}$ \\
\hline
\end{tabular}


Ir ao banheiro - dirige-se ao banheiro para urinar ou evacuar: faz sua higiene e se veste após eliminações

\begin{tabular}{|c|c|c|}
\hline $\begin{array}{l}\text { Vai ao banheiro, higieniza-se e se } \\
\text { veste após as eliminações sem } \\
\text { assistência (pode utilizar objetos de } \\
\text { apoio como bengala, andador, barras } \\
\text { de apoio ou cadeira de rodas e pode } \\
\text { utilizar comadre ou urinol à noite } \\
\text { esvaziando por si mesmo pela } \\
\text { manhã) }\end{array}$ & $\begin{array}{c}\text { Recebe assistência para ir ao } \\
\text { banheiro ou para higienizar-se ou } \\
\text { para vestir-se após as eliminações } \\
\text { ou para usar urinol ou comadre à } \\
\text { noite }\end{array}$ & $\begin{array}{c}\text { Não vai ao banheiro para urinar } \\
\text { ou evacuar }\end{array}$ \\
\hline \multicolumn{3}{|c|}{ Transferência } \\
\hline $\begin{array}{c}\text { Deita-se e levanta-se da cama ou da } \\
\text { cadeira sem assistência (pode utilizar } \\
\text { um objeto de apoio como bengala ou } \\
\text { andador) }\end{array}$ & $\begin{array}{c}\text { Deita-se e levanta-se da cama ou } \\
\text { da cadeira com auxílio }\end{array}$ & Não sai da cama \\
\hline \multicolumn{3}{|c|}{ Continência } \\
\hline $\begin{array}{l}\text { Tem controle sobre as funções de } \\
\text { urinar e evacuar }\end{array}$ & $\begin{array}{l}\text { Tem perdas urinárias ou fecais } \\
\text { ocasionais }\end{array}$ & $\begin{array}{l}\text { Necessita de supervisão para } \\
\text { controlar a urina, fezes, utiliza } \\
\text { cateterismo ou é incontinente }\end{array}$ \\
\hline \multicolumn{3}{|c|}{ Alimentação } \\
\hline Alimenta-se sem assistência & $\begin{array}{l}\text { Alimenta-se sem assistência, } \\
\text { exceto para cortar carne ou passar } \\
\text { manteiga no pão }\end{array}$ & $\begin{array}{c}\text { Recebe assistência para se } \\
\text { alimentar ou é alimentado parcial } \\
\text { ou totalmente por sonda enteral } \\
\text { ou parenteral }\end{array}$ \\
\hline
\end{tabular}

CLASSIFICAÇÃO:

( ) A: Independente em todas as funções

( ) B: Independente em cinco funções

( ) C: Independente em todas exceto lavar-se e outra

( ) D: Independente em todas exceto lavar-se, vestir-se e outra

( ) E: Independente em todas exceto, lavar-se, vestir-se, usar o sanitário e outra

( ) F: Independente em todas exceto lavar-se, vestir-se, usar a sanita, mobilizar-se e outra

( ) G: Dependente em todas as funções

( ) Outro: Dependente em pelo menos duas funções, mas que não se classificasse em C, D, E e F.

\section{Estado nutricional - Nutricionista:}

\begin{tabular}{|l|}
\hline \multicolumn{1}{|c|}{ AVALIAÇÃO NUTRICIONAL } \\
\hline Peso Atual: \\
Altura: \\
IMC: \\
\%CB: \\
CP: \\
Avaliação Nutricional: ( ) Desnutrição Grave ( ) Desnutrição ( ) Eutrófico ( ) Sobrepeso \\
( ) Obesidade
\end{tabular}




\section{CONDUTA NUTRICIONAL}

DIETA:

Via: ( ) Oral ( ) Enteral ( ) Gástrica ( ) Gastrostomia ( ) Parenteral

Tipo: ( ) Hipossódica ( ) Hipoglicidica ( ) Sem sal ( ) Sem gordura ( ) Laxante

( ) Constipante ( )

Consistência: ( ) Livre ( ) Branda ( ) Pastosa ( ) Liquida Pastosa ( ) Líquida Total

( ) Líquida de Prova

Suplemento: $($ )

Simbiótico/Fibra: ( )

\section{Situação sócio-familiar - Assistente Social:}

Constituição familiar no lar:

Escolaridade:

Profissão: Aposentado: ( ) Sim ( ) Não

( ) BPC

Renda familiar: $<1 \mathrm{Sm}(\quad)$ 1-3 Sm ( ) $>3 \mathrm{Sm}(\quad)$

Bolsa Família: ( ) Sim ( ) Não Valor:

Lazer: Sim ( ) Não ( )

Alimentação diária:

Forma de transporte: Ônibus ( ) Carro próprio ( ) Carro do Município ( )

Acompanhamento pela Atenção Básica: Sim ( ) Não ( )

Forma de acompanhamento:

Dados do Cuidador:

Nome:

Parentesco:

Idade:

Data de nascimento:

Sexo: ( ) Masc. ( ) Fem.

Endereço:

Telefone:

Escolaridade: Profissão: Aposentado: ( ) Sim ( ) Não

Estado civil: ( ) Casado(a) ( ) Solteiro(a)( ) Divorciado(a) ( ) Viúvo(a)

17. Planejamento de Saúde: 